\title{
Collision model approach to steering of an open driven qubit
}

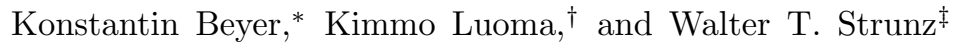 \\ Institut für Theoretische Physik, Technische Universität Dresden, D-01062, Dresden, Germany
}

(Dated: March 23, 2022)

\begin{abstract}
We investigate quantum steering of an open quantum system by measurements on its environment in the framework of collision models. As an example we consider a coherently driven qubit dissipatively coupled to a bath. We construct local non-adaptive and adaptive as well as nonlocal measurement scenarios specifying explicitly the measured observable on the environment. Our approach shows transparently how the conditional evolution of the open system depends on the type of the measurement scenario and the measured observables. These can then be optimized for steering. The nonlocal measurement scenario leads to maximal violation of the used steering inequality at zero temperature. Further, we investigate the robustness of the constructed scenarios against thermal noise. We find generally that steering becomes harder at higher temperatures. Surprisingly, the system can be steered even when bipartite entanglement between the system and individual subenvironments vanishes.
\end{abstract}

\section{INTRODUCTION}

Perhaps the most fascinating feature of quantum theory is the existence of correlations that cannot be explained by classical local randomness. Since the dawn of the quantum age it has been known that entanglement, a term coined by Schrödinger [1, can give rise to paradoxes when seen in the light of a realistic theory as pointed out by EPR 2. About 30 years later, a formulation that allowed experimental testing of nonlocal phenomena of the theory was given by Bell [3]. More recently, Wiseman et.al. discussed another facet of quantum correlations, namely steerability. Indeed, they showed that nonlocality, steerability and entanglement form a strict hierarchy 4. Steering is a task where one party is trying to remotely influence another party's state by local measurements. Over the last decade this phenomenon has been under intensive theoretical [5-13] and experimental [14-17 investigations.

Mostly, steering has been analyzed in situations where quantum dynamics plays no role. In general, quantum systems are coupled to their environment and thus they are open. Let us assume that the open system and the environment both are initially in a pure state. Accordingly, the joint state then evolves as

$$
\left|\Psi_{t}\right\rangle=\mathrm{U}_{t}\left(\left|\psi_{\mathcal{S}}\right\rangle \otimes\left|\psi_{\mathcal{A}}\right\rangle\right)
$$

where $\mathrm{U}_{t}$ is a unitary transformation depending on time $t$ and $\left|\psi_{\mathcal{S}}\right\rangle$ and $\left|\psi_{\mathcal{A}}\right\rangle$ are the initial states of the system and the environment, respectively. The reduced system state is then given by $\rho_{\mathcal{S}}=\operatorname{Tr}_{\mathcal{A}}\left[\left|\Psi_{t}\right\rangle\left\langle\Psi_{t}\right|\right]$, where $\operatorname{Tr}_{\mathcal{A}}$ denotes the partial trace over the environment. While the joint state remains pure for all times, the reduced state becomes mixed due to interactions giving rise to entanglement creation

$$
\operatorname{Tr}\left[\rho_{\mathcal{S}}^{2}\right]<1 \Longleftrightarrow\left|\Psi_{t}\right\rangle \text { is entangled. }
$$

\footnotetext{
* konstantin.beyer@tu-dresden.de

$\dagger$ kimmo.luoma@tu-dresden.de

$\ddagger$ walter.strunz@tu-dresden.de
}

Pure entangled states can always be used for quantum steering tasks [4, 18. Therefore, one may ask whether and how the system can be steered by measurements on its environment. Based on this idea, an experiment for showing that quantum jumps in a coherently driven two-level atom are detector dependent was proposed and investigated in 19 21]. The system under consideration there can be described by the Gorini-KossakowskiSudarshan-Lindblad (GKSL) master equation for resonance fluorescence 22,24

$$
\dot{\rho}_{\mathcal{S}}=\mathcal{L}_{\rho_{\mathcal{S}}}=-i \omega\left[\sigma_{\mathrm{x}}, \rho_{\mathcal{S}}\right]+\gamma \sigma_{-} \rho_{\mathcal{S}} \sigma_{+}-\frac{\gamma}{2}\left\{\rho_{\mathcal{S}}, \sigma_{+} \sigma_{-}\right\}
$$

where $\omega$ is the driving strength and $\gamma$ is the damping rate. Master equations, such as Eq. 3), can be unraveled in many different ways producing ensembles that all represent the same reduced state $\rho_{\mathcal{S}}$ [24 26]. In [1921] different adaptive and non-adaptive photo detection schemes are implemented, corresponding to different unravelings, and it was shown that the produced ensembles are able to violate a steering inequality.

We approach the task of steering an open quantum system using collision models [27 34. In unravelings, the continuous monitoring of the environment results in a stochastic evolution equation describing the open system dynamics. In the framework of collision models, the bath consists of many subenvironments interacting discretely and individually with the open quantum system. The measurements on the environment can thus be implemented explicitly as a sequence of measurements on the subenvironments. In this Article we construct suitable local and nonlocal measurement scenarios on the environment, specifying the measured observables, to achieve steering of an open system described by Eq. (3) in the time-continuous limit. Further, we extend the discussion to the case where the open system is coupled to a thermal bath.

The Article is organized as follows. In Sec. II we briefly review the concepts of collision models, discrete quantum trajectories, measurement scenarios and quantum steer- 
ing. In Sec. III A we apply these concepts to the model system. We show that a monitored collision model is suitable for the description of steering in such a system. Specifically, in Sec. IIIE we provide a concrete realization of a nonlocal measurement scenario, which in the timecontinuous limit leads to a Markovian two-qubit GKSL equation, embedding Eq. (3). In Sec. IV we discuss the thermal case and show that steering is possible even when bipartite entanglement between the system an individual subenvironments vanishes. Lastly, in Sec. V]we conclude.

\section{CONCEPTS}

\section{A. Collision model}

Let $\rho_{\mathcal{S}}$ be the density operator describing the open quantum system $\mathcal{S}$ with Hilbert space $\mathcal{H}_{\mathcal{S}}$. The environment $\mathcal{A}$ of the open system is composed of subenvironments $\mathcal{A}_{i}$ with Hilbert spaces $\mathcal{H}_{\mathcal{A}_{i}}$. In the class of collision models we consider, the open system interacts sequentially and only once with each subenvironment. The coupling between $\mathcal{S}$ and $\mathcal{A}_{i}$ is given by a unitary operator $\mathrm{Q}_{i}$. The reduced system state after $n$ collisions then reads [27]

$$
\rho_{\mathcal{S}}(n)=\operatorname{Tr}_{\mathcal{A}}\left[\mathrm{Q}_{n} \ldots \mathrm{Q}_{1}\left(\rho_{S}(0) \bigotimes_{i=1}^{n} \rho_{\mathcal{A}_{i}}\right) \mathrm{Q}_{1}^{\dagger} \ldots \mathrm{Q}_{n}^{\dagger}\right]
$$

where $\rho_{\mathcal{S}}(0)$ and $\bigotimes_{i=1}^{n} \rho_{\mathcal{A}_{i}}$ are the initial states of open system and environment, respectively. We restrict ourselves here to the case where the subenvironments are initially in a product state. Furthermore, we assume that each unitary $\mathrm{Q}_{i}$ acts nontrivially only on the system $\mathcal{S}$ and the $i$ th subenvironment $\mathcal{A}_{i}$. Therefore, it is possible to trace out the $i$ th subenvironment $\mathcal{A}_{i}$ right after the $i$ th collision, without changing the future evolution of the reduced state and we can write recursively 1

$$
\rho_{\mathcal{S}}(i)=\operatorname{Tr}_{\mathcal{A}_{i}}\left[\mathrm{Q}_{i}\left(\rho_{\mathcal{S}}(i-1) \otimes \rho_{\mathcal{A}_{i}}\right) \mathrm{Q}_{i}^{\dagger}\right]
$$

We clearly see that the $i$ th state $\rho_{\mathcal{S}}(i)$ of the open system depends only on $\mathrm{Q}_{i}, \rho_{\mathcal{S}}(i-1)$ and $\rho_{\mathcal{A}_{i}}$ and not on earlier evolution of the open system. Hence, the evolution of the open system is memoryless. The collision model discussed in our work is shown schematically in Fig. 1.

\footnotetext{
1 Please note that these restrictions are in general not necessary for collision models. Initial entanglement in the environment or couplings between the subenvironments can lead to memory effects and are useful in order to model, for example, non-Markovian dynamics. We refer to 35,40 for further details.
}

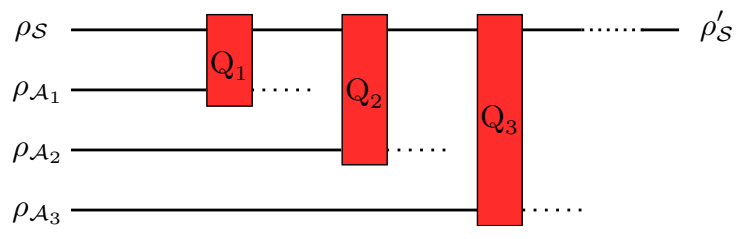

FIG. 1. Scheme of a collision model with the first three collisions between $\mathcal{S}$ and the subenvironments $\mathcal{A}_{i}$. The subenvironments are traced out after their collisions.

\section{B. Discrete quantum trajectories}

Measuring selectively the subenvironments after their interaction with the system — instead of tracing them out - leads to a discrete conditional evolution for $\mathcal{S}$ [41]. While the evolution of the reduced state $\rho_{\mathcal{S}}$ is deterministic, the evolution of conditional states is stochastic, due to the probabilistic nature of quantum measurements.

We denote the joint state of the system and the first subenvironment after their collision by $\rho_{\mathcal{S} \mathcal{A}_{1}}=$ $\mathrm{Q}_{1}\left(\rho_{\mathcal{S}}(0) \otimes \rho_{\mathcal{A}_{1}}\right) Q_{1}^{\dagger}$. The conditional state of the system $\rho_{k \mid E}$ after a measurement on the subenvironment is [42]

$$
\rho_{k \mid E}=\frac{\operatorname{Tr}_{\mathcal{A}_{1}}\left[\rho_{\mathcal{S} \mathcal{A}_{1}}\left(\mathbb{1}_{\mathcal{S}} \otimes E_{k}\right)\right]}{p(k)},
$$

where $k$ labels the measurement outcomes of the positive operator valued measure (POVM) $\left\{E_{k}\right\}$ and $p(k)=$ $\operatorname{Tr}\left[\rho_{\mathcal{S} \mathcal{A}_{1}}\left(\mathbb{1}_{\mathcal{S}} \otimes E_{k}\right)\right]$ is the probability to obtain outcome $k$ when measuring observable $E$. This process can be repeated using $\rho_{k \mid E}$ as the initial system state for the next collision and the subsequent measurement.

In this way a sequence of states $\left\{\rho_{k_{i} \mid E_{i}}\right\}_{i=1}^{N}$, called a discrete quantum trajectory, is obtained. Each $\rho_{k_{i} \mid E_{i}}$ is conditioned on all measurements and their respective outcomes up to and including the $i$ th step. We call the last state $\rho_{k_{N} \mid E_{N}}$ the trajectory endpoint, where $N$ is the number of collisions.

The reduced state after the $i$ th collision $\rho_{\mathcal{S}}(i)$ is recovered by averaging the conditional states at the $i$ th step over all possible trajectories weighted by their probability of occurrence.

\section{Local and nonlocal measurement scenarios}

A set of rules, which determines how the subenvironments are measured, is called a measurement scenario [25].

Local scenario. Let $\mathcal{X}_{i}$ be the set of all possible POVMs on $\mathcal{H}_{\mathcal{A}_{i}}$. For any $X_{i} \in \mathcal{X}_{i}$, let $\Omega_{X_{i}}$ be the set of outcomes. A local measurement scenario for $N$ collisions is a rule how to construct the generalized observable $A \in \mathcal{X}_{1} \times \cdots \times \mathcal{X}_{N}$. Further, the choice of $A$ fixes the outcome space $\Omega_{A}=\Omega_{X_{1}} \times \cdots \times \Omega_{X_{N}}$. We call 
a local measurement scenario non-adaptive if all subenvironments are measured locally and independently of each other. Local scenarios appear as the natural choice for the step-by-step structure of a collision model. Each subenvironment may be measured right after its interaction with the system and can be discarded afterwards (see Fig. 2). In a local non-adaptive measurement scenario, the measured observable $A$ can be predefined before the start of the collision dynamics.

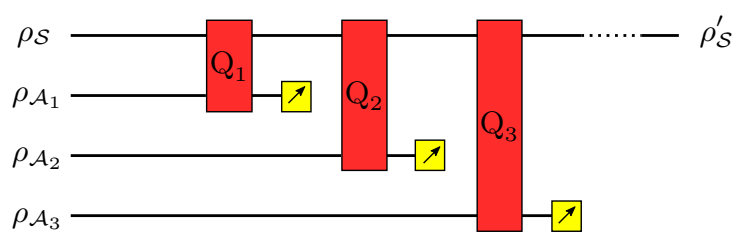

FIG. 2. Scheme of a collision model with local measurements. The open system starts in the state $\rho_{\mathcal{S}}$ and interacts sequentially with the subenvironments which are initially in the states $\rho_{\mathcal{A}_{i}}$. The interaction is described by unitary transformations $\mathrm{Q}_{i}$ and the subenvironments are measured after the interaction.

We can think of a local scenario where the choice of the measurement on the $i$ th subenvironment $\mathcal{A}_{i}$ may depend on previously measured observables $\left\{X_{j}\right\}_{j=1}^{i-1}$ and on their outcomes $\left\{k_{j}\right\}_{j=1}^{i-1}$. A scenario which makes use of this classical information in order to choose the measurement for the next step is called an adaptive measurement scenario [25, 43, 44]. In a local adaptive measurement scenario, the measured observable $A$ is determined during runtime.

Nonlocal scenario. Even though local measurements are directly motivated by the structure of collision models, it is worth considering more general measurements. After $N$ collisions without measurements the joint state of the system and the environment is

$$
\rho_{\mathcal{S} \mathcal{A}_{1} \cdots \mathcal{A}_{N}}=\mathrm{Q}_{N} \cdots \mathrm{Q}_{1}\left(\rho_{\mathcal{S}}(0) \bigotimes_{i=1}^{N} \rho_{\mathcal{A}_{i}}\right) \mathrm{Q}_{1}^{\dagger} \cdots \mathrm{Q}_{N}^{\dagger} .
$$

The most general observable $A^{\text {nonloc }}$ to be measured on the environment can be any from the set of observables on $\mathcal{H}_{\mathcal{A}_{1}} \otimes \cdots \otimes \mathcal{H}_{\mathcal{A}_{N}}$ and is generally nonlocal. In order to measure such a general observable, all of the subenvironments have to be stored after their interaction with the system. Accordingly, we lose the advantages of handling only a few subenvironments instead of the whole environment. For this reason we now introduce a class of nonlocal measurements which is motivated by the structure of collision models and circumvents the storage of many subenvironments.

The measurement scenario is schematically shown in Fig. 3. Instead of being measured after their interaction with the system, the subenvironments are coupled to an ancilla system $\mathcal{C}$, called the control system, which does not belong to the environment but is a part of the measurement apparatus. The interaction is mediated by unitary transformations $\mathrm{T}_{i}$ acting nontrivially on the control system and the respective subenvironment $\mathcal{A}_{i}$. After the T-gate the subenvironments may be traced out. The actual measurement is performed on $\mathcal{C}$ after a sequence of $N$ collisions and the POVM $C=\left\{C_{k}\right\}$ can be any from the set of POVMs on $\mathcal{H}_{\mathcal{C}}$. If the operators $\mathrm{T}_{i}$ are nonlocal unitaries, then the measurement on $\mathcal{C}$ is in general equivalent to some nonlocal measurement on all subenvironments. For a certain POVM element $C_{k}$ we have

$$
p_{k}=\operatorname{Tr}\left[\mathcal{T}_{\mathcal{A C}}\left(\rho_{\mathcal{S} \mathcal{A}_{1} \cdots \mathcal{A}_{N}} \otimes \rho_{\mathcal{C}}\right) \mathcal{T}_{\mathcal{A C}}^{\dagger}\left(\mathbb{1}_{\mathcal{S} \mathcal{A}_{1} \ldots \mathcal{A}_{N}} \otimes C_{k}\right)\right],
$$

where $\mathcal{T}=\mathrm{T}_{N} \circ \ldots \circ \mathrm{T}_{1}$. Therefore, due to the cyclic property of the trace, the nonlocal observable measured by the given measurement scenario is

$$
A_{k}=\operatorname{Tr}_{\mathcal{C}}\left[\mathcal{T}_{\mathcal{A C}}^{\dagger}\left(\mathbb{1}_{\mathcal{A}_{1} \ldots \mathcal{A}_{N}} \otimes C_{k}\right) \mathcal{T}_{\mathcal{A C}}\right]
$$

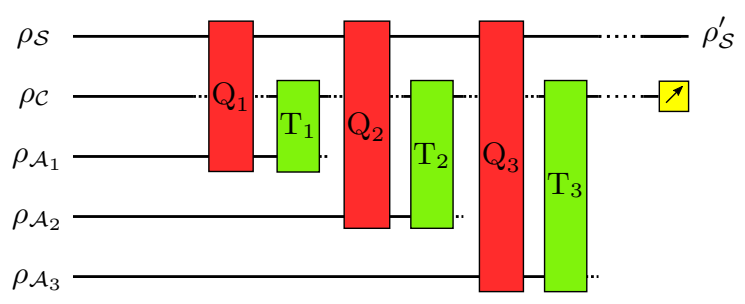

FIG. 3. A collision model which is equivalent to a nonlocal measurement on all involved subenvironments. After its coupling with the system, each subenvironment interacts with the control system by a unitary transformation $\mathrm{T}_{i}$. There is only one measurement in the end performed on the control system $\mathcal{C}$.

It has to be emphasized that in all of these collision models measurements on the environment - no matter to which scenario they may belong - do not change the reduced dynamics of the system described by Eq. (5). This can be most easily understood from Figs. 2 and 3 . where we see that the measurements as well as the couplings $\mathrm{T}_{i}$ to the control system take place after the interactions $\mathrm{Q}_{i}$ between the subenvironments and the open system. Since $\mathcal{S}, \mathcal{A}_{i}$ 's and $\mathcal{C}$ are all initially uncorrelated, the reduced dynamics of $\mathcal{S}$ remains unaffected.

An experimenter who has only access to the system but not to the environment will never be able to determine how the environment is measured.

\section{Quantum steering}

Conditional dynamics of a collision model can be seen as a multipartite steering task. The open system is steered by the measurements on the subenvironments. [4, 19]. For local scenarios the measurements on the first $N$ subenvironments steer the system to the trajectory endpoint. In the nonlocal case the steering is performed by measuring the control system after $N$ collisions.

To formulate the steering scenario, we introduce Alice and Bob. The latter has access to the open system 
$\mathcal{S}$. He can perform arbitrary measurements on his part. Alice cannot directly act on Bob's system but she can perform measurements on the environment, that is on the $\mathcal{A}_{i}$ 's. Note that in the case of a non-adaptive scenario, Alice can measure all subenvironments at once (possibly nonlocally) and conditional dynamics can also be seen as a bipartite steering task. If the measurements on the subenvironments are adaptive, then the scenario corresponds to a multipartite steering task with classical communication between the parties trying to steer.

An important characteristic of any measurement scenario is the set of possible endpoints which the scenario is able to produce. We call this set of states the endpoint ensemble. A single run of the experiment of course only produces a single trajectory with a single (random) endpoint. The endpoint ensemble consists of all possible trajectory endpoints which can be reached using a given measurement scenario. If Alice finds two scenarios which lead to different endpoint ensembles, we have steering in the sense pointed out by Schrödinger already in 1935 [1].

A scenario is said to be steerable if the joint probability distribution for Alice's and Bob's measurement outcomes cannot be written as [4, 5]:

$$
P(a, b \mid A, B)=\sum_{\xi} p(\xi) P_{\mathcal{A}}(a \mid A ; \xi) P_{\mathcal{S}}\left(b \mid B ; \rho_{\xi}\right)
$$

where $A$ and $B$ are the measurements which Alice and Bob perform on their respective parts, $a$ and $b$ are the outcomes, $p$ is a probability distribution and $\xi$ is a local hidden variable. $P_{\mathcal{A}}$ is a local probability distribution which can be arbitrary because Bob does not trust Alice, so she could announce outcomes according to an arbitrary distribution. By contrast, the distribution at Bob's side $P_{\mathcal{S}}$ is a quantum probability distribution because Bob trusts himself that he indeed performs quantum measurements 5 .

Next we discuss how steering is defined in collision models, thus specifying what the observables $A, B$ and their outcomes $a, b$ are. Alice wants to steer the state of the open system after $N$ collisions. Therefore we are interested in the steerability properties of the joint state $\rho_{S \mathcal{A}_{1} \cdots \mathcal{A}_{N}}$ produced by the collision dynamics.

In the local adaptive and non-adaptive measurement scenarios the outcomes $a$ of Alice's observable $A$ are random vectors of length $N$ on the outcome space $\Omega_{A}$.

The restricted class of nonlocal measurement scenarios is equivalent to the usual bipartite steering scenarios, since Alice measures a single observable $A$ on the control system.

It is in general very difficult to proof that a measured probability distribution cannot be decomposed as in Eq. 10. In practice, steering is witnessed by using inequalities which are violated only if Alice is able to steer Bob's system. There are several different forms of such inequalities (see e.g. 4 6] ), depending on the concrete systems involved.

\section{STEERING OF A DRIVEN QUBIT}

\section{A. The model}

In this section we will apply the different types of measurement scenarios to a resonantly driven two-level system coupled to a vacuum bath. We will see that the different measurement scenarios lead to different endpoint ensembles and, furthermore, that these ensembles are indeed able to violate a steering inequality.

The system of interest is a two-level system. The environment consists of qubit subenvironments, initially in a product state $\rho_{\mathcal{A}}=\bigotimes_{i}|0\rangle\langle 0|$, where $|0\rangle$ is the ground state. The interaction between the system and a single subenvironment is given by the unitary transformation

$$
\mathrm{W}_{i}=e^{-i g\left(\sigma_{+} \otimes \sigma_{-}{ }^{i}+\sigma_{-} \otimes \sigma_{+}{ }^{i}\right)}
$$

where $g$ is a real coupling constant [41. The driving is modeled by a local unitary transformation only acting on $\rho_{\mathcal{S}}$

$$
\mathrm{U}=e^{-i f \sigma_{\mathrm{x}}}
$$

where $f$ is a real constant. We set $\mathrm{Q}_{i}=\mathrm{W}_{i} \mathrm{U}$, and define a single step map $\mathcal{E}_{i}: \rho_{\mathcal{S}} \mapsto \mathcal{E}_{i}\left(\rho_{\mathcal{S}}\right)$ for the reduced state using Eq. 5] 36

$$
\mathcal{E}_{i}\left(\rho_{\mathcal{S}}\right)=\operatorname{Tr}_{\mathcal{A}_{i}}\left[\mathrm{Q}_{i}\left(\rho_{\mathcal{S}} \otimes|0\rangle\left\langle\left. 0\right|_{i}\right) \mathrm{Q}_{i}^{\dagger}\right]\right.
$$

Since the collision model is homogeneous the action of the map $\mathcal{E}_{i}$ is the same for any $i$ and we can set $\mathcal{E}_{i}=\mathcal{E}$.

The steady state of the map is $\mathcal{E}\left(\rho_{S S}\right)=\rho_{S S}$ and its Bloch vector reads

$$
\mathbf{r}_{S S}=\left(\begin{array}{c}
x_{S S} \\
y_{S S} \\
z_{S S}
\end{array}\right)=\left(\begin{array}{c}
0 \\
\frac{4 \sin ^{2}\left(\frac{g}{2}\right) \cos (g) \sin (2 f)}{-4 \cos (g) \cos ^{2}(f)+\cos (2 g)+3} \\
\frac{(2-2 \cos (g))(\cos (g) \cos (2 f)-1)}{-4 \cos (g) \cos ^{2}(f)+\cos (2 g)+3}
\end{array}\right) .
$$

It is well known that this collision model can reproduce the master equation (3) in the time-continuous limit 36 , 41. We define $g=\sqrt{\gamma \delta t}$ and $f=\omega \delta t$ and expand the map 13 to first order in $\delta t$. In the limit $\delta t \rightarrow 0$ we then obtain the GKSL master equation (3) describing a damped driven two-level system at zero temperature [24].

\section{B. Steering inequality}

The steering inequality which we use was proposed in 19. It only depends on quantities which can be calculated from the endpoint ensembles which Alice produces after $N$ collisions but not on the full sequence of outcomes. The endpoint ensemble produced by a measurement scenario $Z$ consists of states $\rho_{l}^{Z}$, where $l$ labels the ensemble members with $\sum_{l} p^{Z}(l) \rho_{l}^{Z}=\rho_{\mathcal{S}}(N)$. By $\mathrm{E}_{Z}\left[\left\langle\sigma_{\mathbf{n}}\right\rangle^{2}\right]=\sum_{l} p^{Z}(l)\left\langle\sigma_{\mathbf{n}}(l)\right\rangle^{2}$, with $\left\langle\sigma_{\mathbf{n}}(l)\right\rangle=\operatorname{Tr}\left[\rho_{l}^{Z}(\mathbf{n} \cdot\right.$ $\boldsymbol{\sigma})$ ], we denote the average of the squared spin component $\left\langle\sigma_{\mathbf{n}}\right\rangle^{2}$ in the direction $\mathbf{n}$ over the ensemble produced 
by measurement scenario $Z$. It is important to remember that the states of the endpoint ensemble depend on the full sequence of outcomes.

Quantum steering can be demonstrated if Alice is able to produce three endpoint ensembles $\left\{\rho_{l}^{Z_{1}}\right\},\left\{\rho_{l}^{Z_{2}}\right\}$ and $\left\{\rho_{l}^{Z_{3}}\right\}$ which violate an inequality of the form:

$$
\mathrm{E}_{Z_{1}}\left[\left\langle\sigma_{\mathbf{n}}\right\rangle^{2}\right]+\mathrm{E}_{Z_{2}}\left[\left\langle\sigma_{\mathbf{m}}\right\rangle^{2}\right]+\mathrm{E}_{Z_{3}}\left[\left\langle\sigma_{\mathbf{k}}\right\rangle^{2}\right] \leq 1
$$

with $\mathbf{n} \perp \mathbf{m} \perp \mathbf{k}$. If Alice cannot produce three but only two different ensembles, the inequality can still be violated by setting $Z_{2}=Z_{3}$.

It is inherent in a steering task that Bob does not trust Alice, so we have to describe how Bob can verify that Alice indeed has produced the ensemble she claims and how Bob can calculate the values needed to check inequality 15 .

Alice and Bob perform many runs of the same experiment 19:

1. Bob prepares an initial state $\rho_{\mathcal{S}}$ and tells Alice which ensemble he would like her to produce.

2. They start the experiment and Alice uses a measurement scenario that produces Bob's desired ensemble.

3. After $N \gg 1$ steps Bob stops the interaction with Alice's part and performs a measurement which he chooses randomly from a set of informationally complete observables [45 47.

4. Alice tells Bob which trajectory endpoint she has produced in this run by using her knowledge of the experiment and her measurement outcomes.

5. Bob writes his outcome and the measurement he has performed on a slip of paper and throws it into a bin which is labeled by the endpoint which Alice has told him, and the measurement scenario she has used.

6. They jump back to point 1 and start the next run.

The number of bins Bob needs depends on how many different trajectory endpoints Alice announces during the runs. If the endpoint ensembles which Alice can produce consist of only a few points, then Bob will only have to label bins for these endpoints. In order to check whether Alice indeed produces the states she announces, Bob has to perform quantum tomography for each bin. Accordingly, he has to ensure that he has collected enough entries for each bin. The risk of being cheated by Alice decreases with increasing number of runs. When Bob has stopped the runs, he can reconstruct the endpoint ensembles as follows.

a) For each bin he does quantum tomography using the information on the slips.

b) He sorts all the bins according to the measurement scenarios. c) The reconstructed states belonging to the same measurement scenario form the endpoint ensemble of that scenario.

The reconstructed ensembles should agree with the ensembles that Alice has reported to Bob, if she has been honest ${ }^{2}$. If the steering inequality is violated, Bob can be sure that his system has really interacted and has become entangled with Alice's qubits. Violation also verifies that Alice has not used some hidden stochastic pure state model to produce the trajectories.

In general there are infinitely many possible measurement scenarios. We will consider some examples which lead to very different endpoint ensembles.

\section{Local non-adaptive scenarios}

In the simplest measurement scenario Alice measures the same sharp observable $\mathrm{S}_{\mathbf{n}}^{ \pm}=\frac{1}{2}(\mathbb{1} \pm \mathbf{n} \cdot \boldsymbol{\sigma})$ on each subenvironment [41, 48]. That is, each subenvironment is projected in the same basis after its collision. In Fig. 4 we plot the endpoint ensembles on the Bloch sphere for measurement scenarios where Alice does the spin measurement in $\mathbf{x}-, \mathbf{y}$ - and $\mathbf{z}$-direction, respectively. The parameters used for the simulations are

$$
\gamma=1, \omega=10, \delta t=0.001
$$

with $g=\sqrt{\gamma \delta t}$ and $f=\omega \delta t$. Every point is the endpoint of a single discrete trajectory of length $N=10^{6}$. We plot $10^{3}$ points. Here the system is initially prepared in the steady state of its reduced dynamics but note that in the limit of long trajectories $(l \rightarrow \infty)$ the ensembles do not depend on the initial state.

Different choices for the measurement confine the endpoint ensembles to different regions on the surface of the Bloch sphere. While the $\mathbf{x}$ - and the $\mathbf{z}$-ensemble are located on the great circle around the $x$-axis, the $\mathbf{y}$ ensemble looks different. The trajectory endpoints are spread over the whole Bloch sphere with a higher density close to the poles of the $x$-axis (see Fig. 4 (a-c)).

Since measurements in $\mathbf{x}$ - and $\mathbf{z}$-direction lead to the same ensembles, these scenarios will not violate the steering inequality 15. In contrast, if one of them is combined with the $\mathbf{y}$-ensemble, the steering inequality 15 is violated. We have $\mathrm{E}_{\mathbf{x}}\left[\left\langle\sigma_{\mathbf{y}}\right\rangle^{2}\right]+\mathrm{E}_{\mathbf{x}}\left[\left\langle\sigma_{\mathbf{z}}\right\rangle^{2}\right]=1$ because all points produced by measurements in $\mathbf{x}$-direction are located on the great circle around the $x$-axis. For measurements in $\mathbf{y}$-direction we obtain numerically $\mathrm{E}_{\mathbf{y}}\left[\left\langle\sigma_{\mathbf{x}}\right\rangle^{2}\right]=$ $0.546 \pm 0.002$. Thus, there is a violation.

\footnotetext{
2 There might be a unitary rotation of all ensembles if Bob's and Alice's basis definitions do not agree but this is unimportant for the success of the steering task.
} 

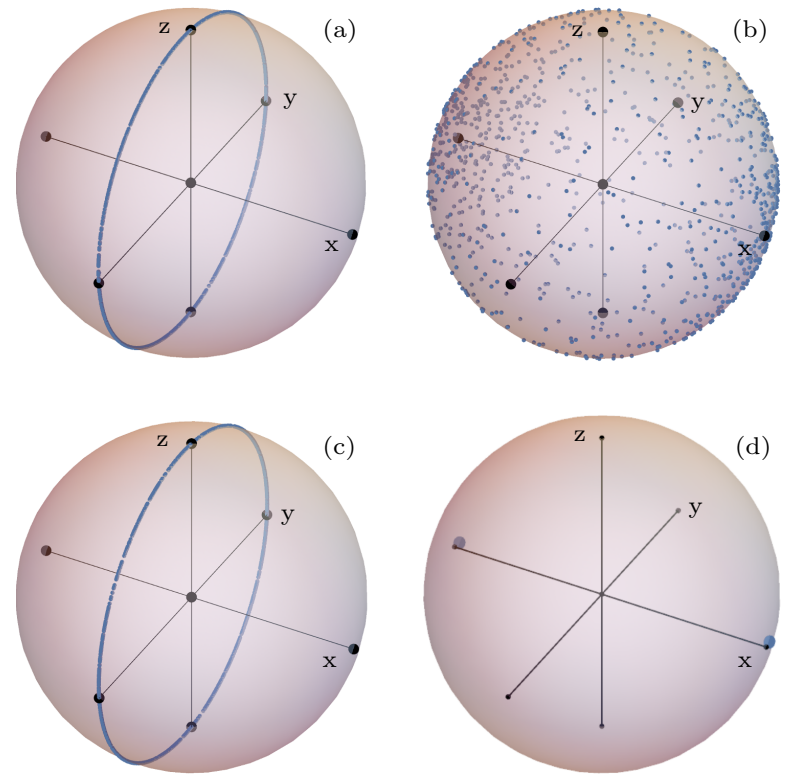

FIG. 4. Endpoint ensembles. Measurements in $\mathbf{x}$ - (a) and $\mathbf{z}$-direction (c) confine the trajectory endpoints to a great circle around the $x$-axis. The $\mathbf{y}$-ensemble (b) is spread over the whole Bloch sphere with a higher density around the $x$ eigenstates. The dichotomic ensemble shown in (d) was obtained from an adaptive scenario. We have plotted $10^{3}$ points for each ensemble. The parameters are given in Eq. 16.

\section{Local adaptive scenario}

The $\mathbf{x}-, \mathbf{y}$ - and $\mathbf{z}$-ensembles are not ideal with respect to the steering inequality (15). A larger violation can be reached if the great-circle-ensemble (produced by measurements in $\mathbf{x}$ - or $\mathbf{z}$-direction) is combined with an ensemble which only consist of points close to the $x$ eigenstates.

If the measurement direction for the subenvironments is not fixed, then it is possible to produce an ensemble which is close to such a dichotomic one 43. The driving rotates the system state around the $x$-axis. Thus, we should look for a dichotomic ensemble whose states are relatively unaffected by the driving, that is, which are close to the $x$-axis. The dichotomic pure state ensemble which satisfies the average reduced steady state $\mathbf{r}_{S S}$ and whose points are as close as possible to the $x$-axis consists of states

$$
\mathbf{r}^{ \pm}=\left(\begin{array}{c} 
\pm \sqrt{1-y_{S S}^{2}-z_{S S}^{2}} \\
y_{S S} \\
z_{S S}
\end{array}\right)
$$

where $y_{S S}$ and $z_{S S}$ are taken from the steady state Bloch vector in Eq. 14.

To have a dichotomic endpoint ensemble, necessarily, as soon as the state of the trajectory is in the ensemble, it can either stay unaffected by the subsequent collision and measurement or jump to the other state in the ensemble.
This leads to the following conditions

$$
\begin{aligned}
& \frac{\operatorname{Tr}_{\mathcal{A}_{i}}\left[\left(\mathbb{1} \otimes \mathrm{S}_{\mathbf{n}_{1}}^{ \pm}\right)\left(\mathrm{Q}_{i}\left(\rho_{\mathcal{S}}^{+} \otimes \rho_{\mathcal{A}_{i}}\right) \mathrm{Q}_{i}^{\dagger}\right)\left(\mathbb{1} \otimes \mathrm{S}_{\mathbf{n}_{1}}^{ \pm}\right)\right]}{p_{\mathbf{n}_{1}}^{ \pm}}=\rho_{\mathcal{S}}^{\mp}, \\
& \frac{\operatorname{Tr}_{\mathcal{A}_{i}}\left[\left(\mathbb{1} \otimes \mathrm{S}_{\mathbf{n}_{2}}^{ \pm}\right)\left(\mathrm{Q}_{i}\left(\rho_{\mathcal{S}}^{-} \otimes \rho_{\mathcal{A}_{i}}\right) \mathrm{Q}_{i}^{\dagger}\right)\left(\mathbb{1} \otimes \mathrm{S}_{\mathbf{n}_{2}}^{ \pm}\right)\right]}{p_{\mathbf{n}_{2}}^{ \pm}}=\rho_{\mathcal{S}}^{ \pm},
\end{aligned}
$$

where $\rho_{\mathcal{S}}^{ \pm}=\frac{1}{2}\left(\mathbb{1}+\mathbf{r}^{ \pm} \cdot \boldsymbol{\sigma}\right), p_{\mathbf{n}_{i}}^{ \pm}$are measurement outcome probabilities and $\boldsymbol{S}_{\mathbf{n}_{1}}$ and $\boldsymbol{S}_{\mathbf{n}_{2}}$ are unknown observables to be found.

The Eqs. $18,(19)$ are satisfied if the directions of the two spin observables are

$$
\mathbf{n}_{1}=\left(\begin{array}{c}
0 \\
\sin (g) \\
\cos (g)
\end{array}\right), \quad \mathbf{n}_{2}=\left(\begin{array}{c}
0 \\
-\sin (g) \\
\cos (g)
\end{array}\right)
$$

The dichotomic ensemble can be produced from any initial state using the following measurement scenario.

1. Alice chooses first to measure the spin in one of the directions $\mathbf{n}_{i}$, arbitrarily.

2. As long as she obtains outcome -1 she keeps measuring to the chosen direction.

3 . When she obtains an outcome +1 she will change to the other direction and continues at step 2.

As shown in Fig. 4(d), this measurement scenario produces the dichotomic ensemble. Note that, as $\delta t \rightarrow 0$, our measurement scenario reduces to the adaptive quantum jump scheme of Ref. [43.

\section{E. Nonlocal scenario}

We turn now to nonlocal scenarios as introduced in Section II B. Let us assume that the system qubit is initially in a pure state (if it is in a mixed state, then we can apply a local measurement scenario until the trajectory has reached the surface of the Bloch ball and, hence, a pure state.). Since all subenvironments are initially in the ground state and all interactions are unitary, the global joint state will remain pure for any number of collisions. In the strong driving limit $\omega \gg \gamma$, the reduced state of the open system is almost maximally mixed $\rho_{S S} \approx \frac{1}{2} \mathbb{1}$. Therefore, in principle, it is possible to steer the system to any dichotomic pure state ensemble if one applies the right global measurement to the whole environment. As already mentioned in Section II B this is not in the spirit of a collision model. Alternatively, we would like to collect the entanglement between the system and the whole environment in a single maximally entangled two-qubit state which would provide the same steering capabilities as the global joint pure state. 
To reach this aim, we add a two dimensional ancilla $\mathcal{C}$ which acts as a control system. After each collision between $\mathcal{S}$ and the subenvironment $\mathcal{A}_{i}$, the control system $\mathcal{C}$ interacts with $\mathcal{A}_{i}$ with a unitary gate $\mathrm{T}_{i}$, after which $\mathcal{A}_{i}$ can be traced out (see Fig. 3). We want to find a universal T-gate $\mathrm{T}_{\mathcal{C A}_{i}}=\mathrm{T}_{\mathcal{C A}}$, such that

$$
\rho_{\mathcal{S C}}^{\star}=\operatorname{Tr}_{\mathcal{A}_{i}}\left[\mathrm{~T}_{\mathcal{C A}_{i}} \mathrm{Q}_{\mathcal{S A}_{i}}\left(\rho_{\mathcal{S C}}^{\star} \otimes \rho_{\mathcal{A}_{i}}\right) \mathrm{Q}_{\mathcal{S} \mathcal{A}_{i}}^{\dagger} \mathrm{T}_{\mathcal{C} \mathcal{A}_{i}}^{\dagger}\right]
$$

where $\rho_{\mathcal{S} \mathcal{C}}^{\star}$ is (i) a pure steady state for $\mathcal{S}+\mathcal{C}$ and therefore (ii) having the maximal amount of entanglement, with respect to the mixedness of $\rho_{S S}$. The subscripts of the unitaries refer to which subsystems they act.

To reduce the degrees of freedom in Eq. 21, we substitute an ansatz of the form $\Theta_{\mathcal{S C}}^{\star}=\left(\begin{array}{cc}1 & \mathbf{c}^{T} \\ \mathbf{r}_{S S} & R\end{array}\right)$ in the Bloch representation for $\rho_{\mathcal{S} \mathcal{C}}^{\star}\left[49\right.$. $\Theta_{\mathcal{S C}}^{\star}$ gives the correct steady state $\mathbf{r}_{S S}$ for the open system. c and $R$ are as

$$
\mathbf{c}=\left(\begin{array}{c}
0 \\
0 \\
\cos \alpha
\end{array}\right), R=\left(\begin{array}{ccc}
0 & \sin \alpha & 0 \\
-\sin \alpha \cos \beta & 0 & -\sin \beta \\
\sin \alpha \sin \beta & 0 & -\cos \beta
\end{array}\right),
$$

where $\alpha=\arccos \left(\left|\mathbf{r}_{S S}\right|\right)$ and $\beta=\arctan \left(\frac{y_{S S}}{z_{S S}}\right)+\pi^{3}$ The above choice for $\mathbf{c}$ and $R$ satisfies the condition (i) and therefore also (ii).

In order to find $\mathrm{T}_{\mathcal{C} \mathcal{A}}$, we evolve the pure state $\rho_{\mathcal{S C}}^{\star}=$ $\left|\psi_{\mathcal{S} \mathcal{C}}^{\star}\right\rangle\left\langle\psi_{\mathcal{S} \mathcal{C}}^{\star}\right|$ over a single collision

$$
\left|\psi_{\mathcal{S C} \mathcal{A}}^{\prime}\right\rangle=\mathrm{Q}\left(\left|\psi_{\mathcal{S C}}^{\star}\right\rangle \otimes|0\rangle\right)
$$

In order to keep $\mathcal{S}+\mathcal{C}$ maximally entangled we have to find a $T_{\mathcal{C A}}$ such that the subenvironment is decoupled from the system and the control, that is

$$
\left(\mathbb{1}_{\mathcal{S}} \otimes \mathrm{T}_{\mathcal{C A}}\right)\left|\psi_{\mathcal{S C} \mathcal{A}}^{\prime}\right\rangle=\left|\psi_{\mathcal{S C}}^{\prime}\right\rangle \otimes\left|0_{\mathcal{A}}\right\rangle
$$

$\mathrm{T}_{\mathcal{C} \mathcal{A}}$ can be constructed by writing $\left|\psi_{\mathcal{S C A}}^{\prime}\right\rangle$ in its Schmidt decomposition

$$
\left|\psi_{\mathcal{S C} \mathcal{A}}^{\prime}\right\rangle=\lambda_{1}\left|\psi_{\mathcal{S}}^{1}\right\rangle \otimes\left|\psi_{\mathcal{C} \mathcal{A}}^{1}\right\rangle+\lambda_{2}\left|\psi_{\mathcal{S}}^{2}\right\rangle \otimes\left|\psi_{\mathcal{C} \mathcal{A}}^{2}\right\rangle
$$

The vectors $\left|\psi_{\mathcal{C} \mathcal{A}}^{1}\right\rangle$ and $\left|\psi_{\mathcal{C} \mathcal{A}}^{2}\right\rangle$ are orthogonal. Together with two further orthogonal vectors $\left|\psi_{\mathcal{C} \mathcal{A}}^{3}\right\rangle$ and $\left|\psi_{\mathcal{C} \mathcal{A}}^{4}\right\rangle$ they form a basis of the two-qubit Hilbert space $\mathcal{H}_{\mathcal{C A}}$. Thus, we can construct the following unitary operator $\mathrm{T}_{\mathcal{C} \mathcal{A}}$ which decouples the subenvironment:

$$
\begin{aligned}
\mathrm{T}_{\mathcal{C} \mathcal{A}}= & \left|0_{\mathcal{C}} 0_{\mathcal{A}}\right\rangle\left\langle\psi_{\mathcal{C} \mathcal{A}}^{1}|+| 1_{C} 0_{A}\right\rangle\left\langle\psi_{\mathcal{C} \mathcal{A}}^{2}\right| \\
& +\left|0_{\mathcal{C}} 1_{\mathcal{A}}\right\rangle\left\langle\psi_{\mathcal{C} \mathcal{A}}^{3}|+| 1_{\mathcal{C}} 1_{\mathcal{A}}\right\rangle\left\langle\psi_{\mathcal{C} \mathcal{A}}^{4}\right|
\end{aligned}
$$

We find

$$
\mathrm{T}_{\mathcal{C A}}=\mathrm{T}_{2} \mathrm{~T}_{1}=e^{-i \omega \delta t S_{2}} e^{-i \sqrt{\gamma \delta t} S_{1}}
$$

\footnotetext{
3 The suitable ansatz was found by considering the given constraints and numerical estimations. This ansatz is not unique but it is chosen such that it leads to a continuous limit.
}

where $S_{1}$ and $S_{2}$ are given in the Appendix A. This unitary $\mathrm{T}_{\mathcal{C} \mathcal{A}}$ together with the $\rho_{\mathcal{S C}}^{\star}$ satisfies Eq. (21).

If Alice uses this nonlocal measurement scenario, she eventually holds one part of a maximally entangled state. As soon as Bob stops the experiment, Alice can steer Bob's system to an arbitrary dichotomic ensemble by performing a projective measurement on the control qubit.

It is possible to obtain a continuous limit for the collision model for $\mathcal{S}+\mathcal{C}$ which leads to a GKSL master equation for the two-qubit state $\rho_{\mathcal{S C}}$. Proceeding as in Sec. III A, the Hamiltonian and the Lindblad operator turn out to be

$$
\begin{aligned}
H_{\mathcal{S C}}= & -\omega\left(\mathbb{1} \otimes \sigma_{\mathrm{y}}\right)+\omega R_{33}\left(\sigma_{\mathrm{x}} \otimes \sigma_{\mathrm{z}}\right)+\omega\left(\sigma_{\mathrm{x}} \otimes \mathbb{1}\right) \\
+ & \frac{1}{4} R_{33} \gamma\left(\sigma_{\mathrm{x}} \otimes \sigma_{\mathrm{x}}\right)+\frac{1}{4} \gamma\left(\sigma_{\mathrm{y}} \otimes \sigma_{\mathrm{y}}\right), \\
L_{\mathcal{S C}}= & -\frac{2 R_{33} \omega}{\sqrt{\gamma}}\left(\mathbb{1} \otimes \sigma_{\mathrm{z}}\right)-\frac{1}{2} R_{33} \sqrt{\gamma}\left(\mathbb{1} \otimes \sigma_{\mathrm{x}}\right) \\
& +\frac{i}{2} \sqrt{\gamma}\left(\mathbb{1} \otimes \sigma_{\mathrm{y}}\right)-i \sqrt{\gamma}\left(\sigma_{-} \otimes \mathbb{1}\right),
\end{aligned}
$$

with $R_{33}=-\frac{\gamma}{\sqrt{\gamma^{2}+16 \omega^{2}}}$.

The GKSL master equation for the open system only, Eq. (3), is embedded into this two-qubit GKSL master equation. Its steady state solution is (in Bloch representation):

$$
\Theta_{S S}=\left(\begin{array}{cccc}
1 & 0 & 0 & \frac{\sqrt{16 c^{2}+1}}{8 c^{2}+1} \\
0 & 0 & \frac{8 c^{2}}{8 c^{2}+1} & 0 \\
\frac{4 c}{8 c^{2}+1} & -\frac{8 c^{2}}{\left(8 c^{2}+1\right) \sqrt{16 c^{2}+1}} & 0 & \frac{4 c}{\sqrt{16 c^{2}+1}} \\
\frac{1}{-8 c^{2}-1} & -\frac{32 c^{3}}{\left(8 c^{2}+1\right) \sqrt{16 c^{2}+1}} & 0 & -\frac{1}{\sqrt{16 c^{2}+1}}
\end{array}\right),
$$

where $c=\omega / \gamma$. Thus, in the continuous limit, this state only depends on the ratio between the driving and the damping.

In the collision model the creation of the entanglement of the bipartite state $\rho_{\mathcal{S C}}$ is mediated by the subenvironments and the control and the system never interact directly. Interestingly, in the continuous limit the Hamiltonian $H_{\mathcal{S C}}$ is of interaction type.

\section{COUPLING TO A THERMAL BATH}

We consider now the general case where the environment is allowed to be initially in a mixed state. The question whether the system is entangled with the environment cannot be answered by the simple argument which holds for pure states only. In the collision model we replace the pure initial state of the subenvironments with a thermal one $\rho_{\mathcal{A}}=\bigotimes_{i} \frac{1}{2}\left(\mathbb{1}^{i}+\eta \sigma_{\mathrm{z}}{ }^{i}\right)$, where $\eta \in(-1,0)$ is a temperature parameter, related to the Boltzmann factor 30. The collision model now describes a damped driven qubit coupled to a thermal bath. Correspondingly, 
the time-continuous limit of our model gives the GKSL master equation

$$
\dot{\rho}_{\mathcal{S}}=-i \omega\left[\sigma_{\mathrm{x}}, \rho_{\mathcal{S}}\right]+\gamma \frac{1-\eta}{2} \mathcal{D}\left[\sigma_{-}\right] \rho_{\mathcal{S}}+\gamma \frac{1+\eta}{2} \mathcal{D}\left[\sigma_{+}\right] \rho_{\mathcal{S}},
$$

where $\mathcal{D}[L] \rho=L \rho L^{\dagger}-\frac{1}{2}\left\{\rho, L^{\dagger} L\right\}$.

The ensembles produced by any measurement scenario in a collision model with thermal subenvironments will certainly not be pure. On the other hand, the steering inequality (15) does not rely on pure ensembles but only on the ensemble averages for $\left\langle\sigma_{\mathbf{n}}\right\rangle^{2}$. In Fig. 5 we show endpoint ensembles for measurements in $\mathbf{x}-,, \mathbf{y}-$ and $\mathbf{z}-$ direction, respectively, and for the adaptive scenario for a temperature $\eta=-0.9$. The parameters are chosen again as in Eq. (16).
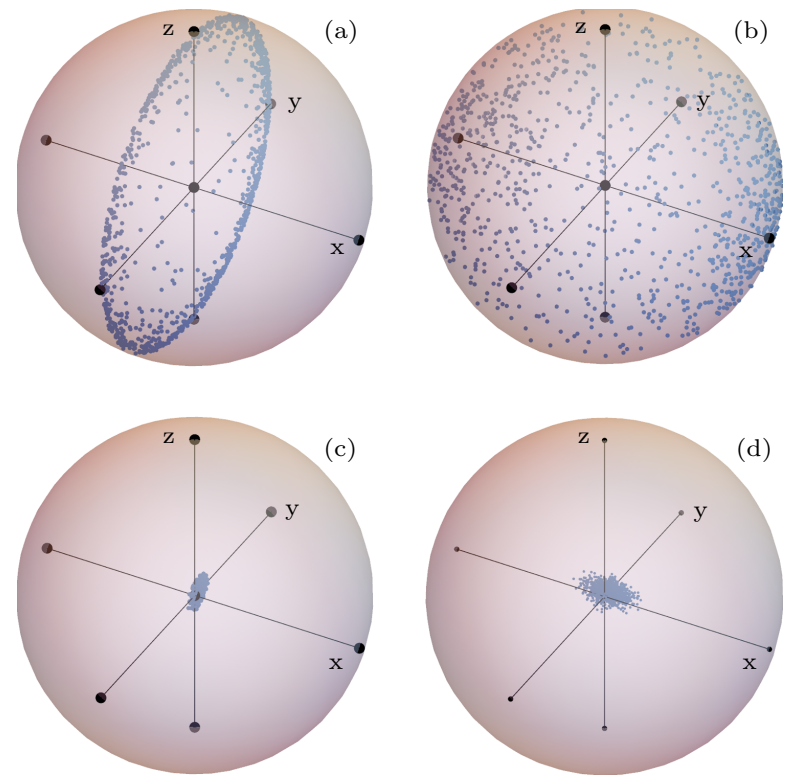

FIG. 5. If the subenvironments are in a thermal state, the endpoint ensembles are not pure anymore. For measurements in $\mathbf{x}$ - and $\mathbf{y}$-direction $(\mathrm{a}, \mathrm{b})$ the ensembles resemble the ensembles obtained in the vacuum case. By contrast, measurements in $\mathbf{z}$-direction (c) as well as the adaptive scenario (d) lead to ensembles which are concentrated close to the center of the Bloch sphere, that is the fully mixed state. The ensembles (c) and (d) cannot demonstrate steering but (a) and (b) violate a steering inequality if the temperature is not too high.

We see that some measurement scenarios are more robust against thermal noise than others. In all cases the thermal subenvironments lead to mixed conditional states in Bob's system, since Alice does not fully know the state of her system before the collisions. While the purity of the conditional states in the $\mathbf{x}$ - or $\mathbf{y}$-ensemble decreases slowly with increasing temperature, the conditional states in the $\mathbf{z}$ - and in the adaptive ensemble lose their purity even if the temperature is very low. Accordingly, the $\mathbf{x}$ - and $\mathbf{y}$-ensembles are most suitable in order to demonstrate steering. We define the steerability $\Delta S$ :

$$
\Delta S=\mathrm{E}_{\mathbf{x}}\left[\left\langle\sigma_{\mathbf{y}}\right\rangle^{2}\right]+\mathrm{E}_{\mathbf{x}}\left[\left\langle\sigma_{\mathbf{z}}\right\rangle^{2}\right]+\mathrm{E}_{\mathbf{y}}\left[\left\langle\sigma_{\mathbf{x}}\right\rangle^{2}\right]-1 .
$$

Steering is successfully demonstrated whenever $\Delta S>$ 0 . The steerability $\Delta S$ decreases with increasing temperature. This is not surprising because the thermal subenvironments induce noise. Numerical simulations show that there is a critical value $\eta_{\text {crit }}=-0.72 \pm 0.01$ up to which the system can be steered by the chosen measurement scenarios. This value is an upper bound for a wide range of the parameters $\delta t, \omega$ and $\gamma$ in the discrete model. In particular, this $\eta_{\text {crit }}$ is also reached for strong driving $\omega \gg \gamma$ and small time steps $\omega \delta t \ll 1$, where the discrete collision model is a good numerical approximation of the related continuous process. However, we recall that $\eta_{\text {crit }}$ depends on the chosen measurement scenarios and therefore must not be seen as a universal bound for steerability of the system. There might be better measurement scenarios and inequalities which are able to detect steering at even higher temperatures.

Steerability implies entanglement, therefore, at least up to this critical temperature the system and the environment build up entanglement during the interaction. However, we would like to emphasize that there is a big difference between the pure state case (coupling to a vacuum bath) and the thermal case presented in this section. The single pure subenvironments entangle with the system during their collision, whereas the thermal subenvironments do not. More precisely, the two-qubit entanglement between the system and a single subenvironment right after their interaction vanishes in general if the subenvironment is in a thermal state but is nonzero if the subenvironment is initially in a pure vacuum state. Let us assume that the system starts in its steady state $\rho_{S S}$ and, thus, the reduced state does not change during the collisions. Then the bipartite state of the system and a certain subenvironment $\mathcal{A}_{i}$ right after their collision is the same for all $i$. Collisions happening after the interaction between $\mathcal{S}$ and $\mathcal{A}_{i}$ cannot increase the entanglement between these two qubits. Therefore, we only have to check whether the bipartite state $\rho_{\mathcal{S} \mathcal{A}_{i}}^{S S}=Q_{i}\left(\rho_{S S} \otimes \rho_{\mathcal{A}_{i}}\right) \mathrm{Q}_{i}^{\dagger}$ is entangled. The steady state $\rho_{S S}$ depends on the temperature parameter $\eta$ and the time step $\delta t$ and so does the bipartite entanglement 4 . The Bloch vector corresponding to $\rho_{S S}$ can be found in the appendix. In Fig. 6 we show the two-qubit entanglement between the system and single subenvironments for different temperatures and time steps.

For finite time steps $\delta t$ the system always builds up two-qubit entanglement with vacuum subenvironments $(\eta=-1)$. If the bath is thermal, then the temperature up to which bipartite entanglement occurs depends on the time step. The smaller the time step the smaller the temperature $\eta$ above which the two-qubit entanglement

\footnotetext{
${ }^{4}$ We set $\gamma=1$ since it defines only a scaling of $\delta t$ in the discrete model. Then the steady state also depends on the driving parameter $\omega$. However, its influence on the bipartite entanglement is negligible over a wide parameter range. All numerical results presented here are calculated for a fixed driving $\omega=10$.
} 


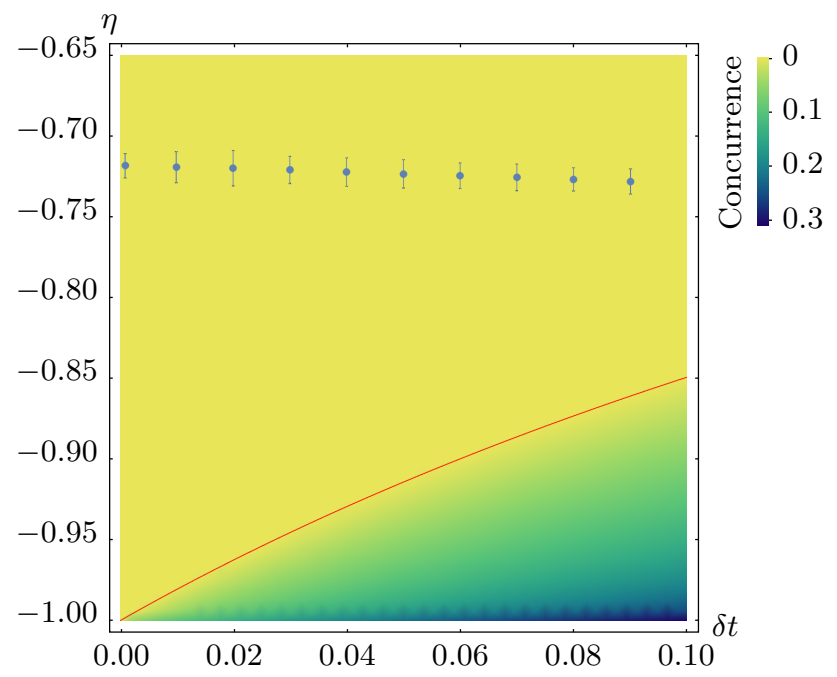

FIG. 6. We plot the concurrence between the system $\mathcal{S}$ and a single subenvironment $\mathcal{A}_{i}$ after their collision. $\mathcal{S}$ is initially prepared in the steady state $\rho_{S S}$ and $\mathcal{A}_{i}$ in the thermal state $\rho_{\mathcal{A}_{i}}=\frac{1}{2}\left(\mathbb{1}+\eta \sigma_{\mathrm{z}}\right)$. The bipartite entanglement depends on the temperature parameter $\eta$ and the time step $\delta t$. In the parameter region beneath the thin line the system becomes entangled with single subenvironments, in the region above it does not. In particular the critical temperatures for steering $\eta_{\text {crit }}$ (blue dots) are in the parameter region without bipartite entanglement.

vanishes. In particular the critical temperature $\eta_{\text {crit }}$ for the steering task is much higher then the temperature which allows bipartite entanglement. The possibility of steering in the thermal case therefore implies that the environment as a whole entangles to the system. It would be surprising if a nonlocal measurement scenario, as seen in Sec. IIIE, which is based on accumulating two-qubit entanglement, could be constructed for the thermal case.

\section{CONCLUSION}

We have shown how the concept of quantum steering of an open quantum system can be discussed elegantly in collision models. The approach provides transparent insights for the choice of actual measurements for the steering tasks. We have argued that local non-adaptive and nonlocal measurement scenarios are instances of bipartite steering, whereas a local adaptive measurement scenario can be seen as a multipartite steering task.

With the help of a concrete example - a coherently driven qubit damped by a bath - we have shown how different measurement scenarios affect the system and how possible endpoint ensembles look like. We have introduced a special form of nonlocal measurements on the environment which fits naturally in the framework of a collision model. In the case of a vacuum bath, such a scenario can be used to accumulate entanglement between the system and the control state. In the time-continuous limit this discrete model leads to a Markovian master equation for two qubits, embedding the reduced system dynamics.

In Sec. IV we have shown that steering is not restricted to the pure state case only, but can also be demonstrated if the environment is in a thermal, that is a mixed, initial state. The robustness against this thermal noise strongly depends on the used measurement scenarios and steering becomes harder with increasing temperature. For two simple scenarios we could numerically estimate a critical value up to which steering can be witnessed. In this temperature range the system and its environment evolve towards an entangled state. Interestingly, there is no two-qubit entanglement between the system and single subenvironments in the thermal case. This leads to the conclusion that steering is based on entanglement between the open system and the whole bath. For deeper insights in how the system gets entangled with its environment it would be necessary to investigate $n$-qubit entanglement in the global joint state which is in general quite involved, both analytically and numerically.

\section{ACKNOWLEDGMENTS}

The authors would like to thank Ana Costa, Chau Nguyen and Roope Uola for many fruitful discussions. We are also grateful to Howard Wiseman for helpful comments on the manuscript.

\section{Appendix A: Universal T-gate}

The exact expressions for the matrices $S_{1}$ and $S_{2}$ in the decomposition for the T-gate in Eq. (27) expressed in the computational basis $\{|11\rangle,|10\rangle,|01\rangle,|00\rangle\}$ are 


$$
\begin{aligned}
S_{1} & =\left(\begin{array}{cccc}
0 & -\frac{2 i R_{33} \omega}{\gamma} & 0 & -\frac{1}{2} i\left(R_{33}-1\right) \\
\frac{2 i R_{33} \omega}{\gamma} & 0 & \frac{1}{2} i\left(R_{33}+1\right) & 0 \\
0 & -\frac{1}{2} i\left(R_{33}+1\right) & 0 & \frac{2 i R_{33} \omega}{\gamma} \\
\frac{1}{2} i\left(R_{33}-1\right) & 0 & -\frac{2 i R_{33} \omega}{\gamma} & 0
\end{array}\right) \\
S_{2} & =\left(\begin{array}{cccc}
0 & 0 & i R_{33} & 0 \\
0 & 0 & 0 & i \\
-i R_{33} & 0 & 0 & 0 \\
0 & -i & 0 & 0
\end{array}\right),
\end{aligned}
$$

\section{Appendix B: Steady state Bloch vector for a thermal environment}

$$
\mathbf{r}_{S S}=\left(\begin{array}{c}
0 \\
-\frac{4 \eta \sin ^{2}\left(\frac{\sqrt{\gamma \delta t}}{2}\right) \cos (\sqrt{\gamma \delta t}) \sin (2 \delta t \omega)}{-4 \cos (\sqrt{\gamma \delta t}) \cos ^{2}(\delta t \omega)+\cos (2 \sqrt{\gamma \delta t})+3} \\
-\frac{4 \eta \sin ^{2}\left(\frac{\sqrt{\gamma \delta t}}{2}\right)(\cos (\sqrt{\gamma \delta t}) \cos (2 \delta t \omega)-1)}{-4 \cos (\sqrt{\gamma \delta t}) \cos ^{2}(\delta t \omega)+\cos (2 \sqrt{\gamma \delta t})+3}
\end{array}\right)
$$

[1] E. Schrödinger, Proceedings of the Cambridge Philosophical Society 31, 555 (1935).

[2] A. Einstein, B. Podolsky, and N. Rosen, Phys. Rev. 47, 777 (1935)

[3] J. S. Bell, Physics 1, 195 (1964).

[4] H. M. Wiseman, S. J. Jones, and A. C. Doherty, Phys. Rev. Lett. 98, 140402 (2007).

[5] S. J. Jones, H. M. Wiseman, and A. C. Doherty, Phys. Rev. A 76, 052116 (2007).

[6] E. G. Cavalcanti, S. J. Jones, H. M. Wiseman, and M. D. Reid, Phys. Rev. A 80, 032112 (2009).

[7] A. C. S. Costa and R. M. Angelo, Phys. Rev. A 93, $020103(2016)$

[8] A. Costa, M. Beims, and R. Angelo, Physica A: Statistical Mechanics and its Applications 461, 469 (2016).

[9] H. C. Nguyen and T. Vu, Phys. Rev. A 94, 012114 (2016)

[10] R. Uola, C. Budroni, O. Gühne, and J.-P. Pellonpää, Phys. Rev. Lett. 115, 230402 (2015).

[11] R. Uola, T. Moroder, and O. Gühne, Phys. Rev. Lett. 113, 160403 (2014)

[12] P. Skrzypczyk, M. Navascués, and D. Cavalcanti, Phys. Rev. Lett. 112, 180404 (2014)

[13] M. T. Quintino, T. Vértesi, and N. Brunner, Phys. Rev. Lett. 113, 160402 (2014).

[14] D. J. Saunders, G. J. Pryde, H. M. Wiseman, and S. J. Jones, Nature Physics 6, 845 (2010)

[15] B. Wittmann, S. Ramelow, F. Steinlechner, N. K. Langford, N. Brunner, H. M. Wiseman, R. Ursin, and A. Zeilinger, New Journal of Physics 14, 053030 (2012).

[16] S. Wollmann, N. Walk, A. J. Bennet, H. M. Wiseman, and G. J. Pryde, Phys. Rev. Lett. 116, 160403 (2016).

[17] Y. Xiao, X.-J. Ye, K. Sun, J.-S. Xu, C.-F. Li, and G.-C. Guo, Phys. Rev. Lett. 118, 140404 (2017).

[18] N. Gisin, Physics Letters A 154, 201 (1991).

[19] H. M. Wiseman and J. M. Gambetta, Phys. Rev. Lett. 108, $220402(2012)$

[20] S. Daryanoosh and H. M. Wiseman, New Journal of Physics 16, 063028 (2014).

[21] S. Daryanoosh, H. M. Wiseman, and J. M. Gambetta, Physical Review A 92, 042114 (2015)

[22] V. Gorini, A. Kossakowski, and E. C. G. Sudarshan, Journal of Mathematical Physics 17, 821 (1976)

[23] G. Lindblad, Communications in Mathematical Physics 48, 119 (1976)

[24] H. Carmichael, An open systems approach to quantum optics, Lecture Notes in Physics Monographs (Springer, Berlin, 1993).

[25] H. M. Wiseman and G. J. Milburn, Quantum Measurement and Control (Cambridge University Press, Leiden, 2009).

[26] H. M. Wiseman and Z. Brady, Phys. Rev. A 62, 023805 (2000).

[27] M. Ziman, P. Štelmachovič, and V. Bužek, Open Systems \& Information Dynamics 12, 81 (2005).

[28] M. Ziman and V. Bužek, Phys. Rev. A 72, 022110 (2005).

[29] M. Ziman, P. Štelmachovič, V. Bužek, M. Hillery, V. Scarani, and N. Gisin, Phys. Rev. A 65, 042105 (2002).

[30] V. Scarani, M. Ziman, P. Štelmachovič, N. Gisin, and V. Bužek, Phys. Rev. Lett. 88, 097905 (2002). 
[31] V. Giovannetti and G. M. Palma, Journal of Physics B: Atomic, Molecular and Optical Physics 45, 154003 (2012)

[32] V. Giovannetti and G. M. Palma, Phys. Rev. Lett. 108, $040401(2012)$

[33] S. N. Filippov, J. Piilo, S. Maniscalco, and M. Ziman, Phys. Rev. A 96, 032111 (2017).

[34] S. Lorenzo, F. Ciccarello, and G. M. Palma, Phys. Rev. A 96, 032107 (2017)

[35] S. Lorenzo, F. Ciccarello, and G. M. Palma, Phys. Rev. A 93, 052111 (2016)

[36] S. Kretschmer, K. Luoma, and W. T. Strunz, Phys. Rev. A 94, 012106 (2016)

[37] K. Luoma, P. Haikka, and J. Piilo, Phys. Rev. A 90, $054101(2014)$

[38] T. Rybár, S. N. Filippov, M. Ziman, and V. Bužek, Journal of Physics B: Atomic, Molecular and Optical Physics 45, 154006 (2012).

[39] F. Ciccarello, G. M. Palma, and V. Giovannetti, Phys. Rev. A 87, 040103 (2013).
[40] B. Çakmak, M. Pezzutto, M. Paternostro, and O. E. Müstecaplığlu, Phys. Rev. A 96, 022109 (2017).

[41] T. A. Brun, American Journal of Physics 70, 719 (2002).

[42] M. Nielsen and I. Chuang, Quantum Computation and Quantum Information, Cambridge Series on Information and the Natural Sciences (Cambridge University Press, 2000).

[43] H. M. Wiseman and G. E. Toombes, Physical Review A 60, 2474 (1999)

[44] R. I. Karasik and H. M. Wiseman, Physical Review Letters 106, 020406 (2011).

[45] G. Mauro D'Ariano, M. G. A. Paris, and M. F. Sacchi, arXiv:quant-ph/0302028 (2003).

[46] C. Carmeli, T. Heinosaari, and A. Toigo, Phys. Rev. A 85, 012109 (2012).

[47] U. Fano, Rev. Mod. Phys. 29, 74 (1957).

[48] P. Busch, P. Lahti, and P. Mittelstaedt, The Quantum Theory of Measurement, Lecture Notes in Physics Monographs (Springer Berlin Heidelberg, 2013).

[49] O. Gamel, Phys. Rev. A 93, 062320 (2016) 